\title{
Fixing Non-determinism
}

\author{
Alexander Vandenbroucke $\quad$ Tom Schrijvers $\quad$ Frank Piessens \\ \{alexander.vandenbroucke, tom.schrijvers, frank.piessens\}@kuleuven.be \\ Department of Computer Science \\ KU Leuven
}

\begin{abstract}
Non-deterministic computations are conventionally modelled by lists of their outcomes. This approach provides a concise declarative description of certain problems, as well as a way of generically solving such problems.

However, the traditional approach falls short when the non-deterministic problem is allowed to be recursive: the recursive problem may have infinitely many outcomes, giving rise to an infinite list.

Yet there are usually only finitely many distinct relevant results. This paper shows that this set of interesting results corresponds to a least fixed point. We provide an implementation based on algebraic effect handlers to compute such least fixed points in a finite amount of time, thereby allowing non-determinism and recursion to meaningfully co-occur in a single program.
\end{abstract}

\section{CCS Concepts}

-Software and its engineering $\rightarrow$ Functional languages; Recursion;

\section{Keywords}

Haskell, Tabling, Effect Handlers, Logic Programming, Non-determinism, Least Fixed Point

\section{INTRODUCTION}

Non-determinism [24] models a variety of problems in a declarative fashion, especially those problems where the solution depends on the exploration of different choices. The conventional approach represents non-determinism as lists of possible outcomes. For instance, consider the semantics of the following non-deterministic expression:

$$
1 ? 2
$$

This expression represents a non-deterministic choice (with the operator ?) between 1 and 2 . Traditionally we model this with the list $[1,2]$. Now consider the next example:

$$
\begin{aligned}
& \operatorname{swap}(m, n)=(n, m) \\
& \text { pair }=(1,2) ? \text { swap pair }
\end{aligned}
$$

Permission to make digital or hard copies of all or part of this work for personal or classroom use is granted without fee provided that copies are not made or distributed for profit or commercial advantage and that copies bear this notice and the full citation on the first page. Copyrights for components of this work owned by others than ACM must be honored. Abstracting with credit is permitted. To copy otherwise, or republish, to post on servers or to redistribute to lists, requires prior specific permission and/or a fee. Request permissions from permissions@ acm.org.

(C) 2016 ACM. ISBN 978-1-4503-2138-9.

DOI: $10.1145 / 1235$
The corresponding Haskell code is:

$$
\begin{aligned}
& \text { swap }::[(a, b)] \rightarrow[(b, a)] \\
& \text { swap } e=[(m, n) \mid(n, m) \leftarrow e] \\
& \text { pair }::[(\text { Int }, \text { Int })] \\
& \text { pair }=[(1,2)]+\text { swap pair }
\end{aligned}
$$

This is an executable model (we use $\ggg$ to denote the prompt of the GHCi Haskell REPL):

$\ggg$ pair

$[(1,2),(2,1),(1,2),(2,1) \ldots$

We get an infinite list, although only two distinct outcomes $((1,2)$ and $(2,1))$ exist. The conventional list-based approach is clearly inadequate in this example. In this paper we model non-determinism with sets of values instead, such that duplicates are implicitly removed. The expected model of pair is then the set $\{(1,2),(2,1)\}$. We can execute this model: ${ }^{1}$

$$
\begin{aligned}
& \text { swap :: (Ord } a, \text { Ord } b) \Rightarrow \operatorname{Set}(a, b) \rightarrow \operatorname{Set}(b, a) \\
& \text { swap }=\operatorname{map}(\lambda(m, n) \rightarrow(n, m)) \\
& \text { pair :: Set (Int, Int) } \\
& \text { pair }=\text { singleton }(1,2) \text { 'union' swap pair } \\
& \ggg \text { pair } \\
& \text { fromList } * * * \text { Exception: <loop> }
\end{aligned}
$$

Haskell lazily prints the first part of the Set constructor, and then has to compute union infinitely many times. As an executable model of non-determinism it clearly remains inadequate: it fails to compute the solution $\{(1,2),(2,1)\}$.

This paper solves the problem caused by the co-occurence of non-determinism and recursion, by recasting it as the least fixed point problem of a different function. The least fixed point is computed explicitly by iteration, instead of implicitly by Haskell's recursive functions.

The contributions of this paper are:

- We define a monadic model that captures both non-determinism and recursion. This yields a finite representation of recursive non-deterministic expressions. We use this representation as a light-weight (for the programmer) embedded Domain Specific Language to build non-deterministic expressions in Haskell.

- We give a denotational semantics of the model in terms of the least fixed point of a semantic function $\mathcal{R} \llbracket \cdot \rrbracket$. The semantics is subsequently implemented as a Haskell function that interprets the model.

- We generalize the denotational semantics to arbitrary complete lattices. We illustrate the added power on a simple

\footnotetext{
${ }^{1}$ Here map comes from Data. Set
} 
graph problem, which could not be solved with the more specific semantics.

- We provide a set of benchmarks to demonstrate the expressivity of our approach and evaluate the performance of our implementation.

\section{OVERVIEW}

\subsection{Non-determinism}

A computation is non-deterministic if it has several possible outcomes. In this paper, we interpret such non-deterministic computations as the set of their results.

Consider the following non-deterministic expression that produces either 1 or 2 :

$$
1 ? 2
$$

For this example, the semantics is given by the set $\{1,2\}$. The semantic function $\llbracket \cdot \rrbracket$ formally characterizes this interpretation:

$$
\begin{aligned}
\llbracket n \rrbracket & =\{n\} \\
\llbracket e_{1} ? e_{2} \rrbracket & =\llbracket e_{1} \rrbracket \cup \llbracket e_{2} \rrbracket
\end{aligned}
$$

The semantics of a literal $n$ is the singleton of $n$, and the semantics of a choice $e_{1} ? e_{2}$ is the union of the semantics of the left and right branches. A simple calculation shows that $\llbracket 1$ ? $2 \rrbracket$ is indeed $\{1,2\}$.

$$
\llbracket 1 ? 2 \rrbracket=\llbracket 1 \rrbracket \cup \llbracket 2 \rrbracket=\{1\} \cup\{2\}=\{1,2\}
$$

Let us extend our semantics to allow for addition of non-deterministic values:

$$
\llbracket e_{1}+e_{2} \rrbracket=\left\{n+m \mid n \in \llbracket e_{1} \rrbracket, m \in \llbracket e_{2} \rrbracket\right\}
$$

Now, consider the expression:

$$
(1 ? 2)+(1 ? 2)
$$

Here we have an expression that contains an addition of two choices. This expression has 4 possible outcomes of which two coincide: the result is either $2(1+1), 3(1+2$ or $2+1)$ or $4(2+2){ }^{2}$ Again calculation gives the expected result:

$$
\begin{aligned}
& \llbracket(1 ? 2)+(1 ? 2) \rrbracket \\
& =\{n+m \mid n \in \llbracket 1 ? 2 \rrbracket, m \in \llbracket 1 ? 2 \rrbracket\} \\
& =\{n+m \mid n \in\{1,2\}, m \in\{1,2\}\} \\
& =\{2,3,4\}
\end{aligned}
$$

Recursion The next example presents a recursive non-deterministic expression pair which chooses between $(1,2)$ and the new primitive swap to swap pair's components around.

$$
\text { pair }=(1,2) ? \text { swap pair }
$$

The semantics of swap $e$ is the set obtained by flipping all pairs in the set of results of $e$ :

$$
\llbracket \text { swap } e \rrbracket=\{(m, n) \mid(n, m) \in \llbracket e \rrbracket\}
$$

The semantics of pair are given by the following recursive equation:

$$
\begin{aligned}
\llbracket \text { pair } \rrbracket & =\llbracket(1,2) ? \text { swap pair } \rrbracket \\
\Longleftrightarrow \llbracket \text { pair } \rrbracket & =\llbracket(1,2) \rrbracket \cup \llbracket \text { swap pair } \rrbracket \\
\Longleftrightarrow \llbracket \text { pair } \rrbracket & =\{(1,2)\} \cup\{(m, n) \mid(n, m) \in \llbracket \text { pair } \rrbracket\}
\end{aligned}
$$

\footnotetext{
${ }^{2}$ The conventional list-based semantics would be $[2,3,3,4]$.
}

This equation admits infinitely many solutions, e.g.

$$
\begin{aligned}
& \llbracket \text { pair } \rrbracket=\{(1,2),(2,1)\}, \\
& \llbracket \text { pair } \rrbracket=\{(0,0),(1,2),(2,1)\}, \\
& \llbracket \text { pair } \rrbracket=\{(1,1),(1,2),(2,1)\}, \\
& \ldots
\end{aligned}
$$

However, we can identify a least solution: the set $\{(1,2),(2,1)\}$ is contained in every other solution.

As we saw previously, a naive translation of this idea in Haskell does not work:

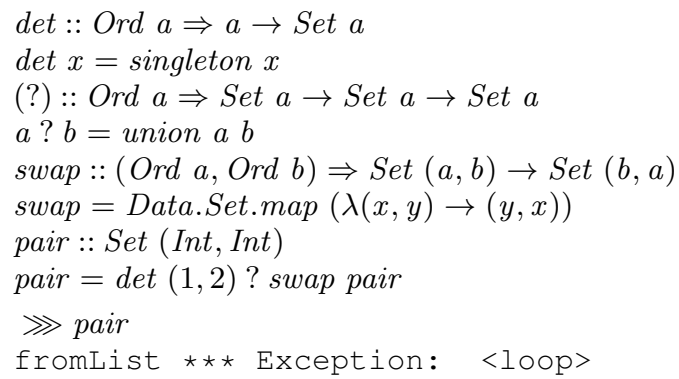

The reason it does not work is that under Haskell's (cpo-based) semantics the equation (1) has a different least solution: $\perp$ (i.e. non-termination). ${ }^{3}$ As this additional $\perp$ in the domain is clearly not the desired solution, we cannot rely on Haskell's native semantics for recursion.

The main contribution of this paper is to reformulate the problem as a different least fixed point problem, for which we can iteratively compute the solution. Moreover, our approach incurs minimal overhead for the programmer, compared to writing the function using the conventional recursive approach.

\subsection{Effect Handlers}

Monads are a way to model side-effects such as non-determinism in a pure functional programming language [25]. In this paper, we use effect handlers to construct a monad for non-determinism and recursion. Effect handlers [10, 27] factor the problem of modeling effectful computations into two parts: first a syntax is introduced to represent all relevant operations, second effect handlers are defined that interpret the syntax within a semantic domain.

The syntax of non-deterministic computations can be modeled with the following data type $N D$, which supports three operations: Success $_{N D} a$ is a deterministic computation with result $a$, Fail is a failed computation, and $O r l r$ represents a non-deterministic choice between two non-deterministic computations $l$ and $r$.

$$
\begin{aligned}
& \text { data } N D a \\
& =\text { Success }_{N D} a \\
& \mid \operatorname{Fail}_{N D} \\
& \mid \operatorname{Or}_{N D}\left(\begin{array}{ll}
N D & a
\end{array}\right)\left(\begin{array}{ll}
N D & a
\end{array}\right)
\end{aligned}
$$

Because the above data type is a free monad [20] we can easily define the following Monad instance:

$$
\begin{aligned}
& \text { instance Monad ND where } \\
& \text { return } a=\text { Success }_{N D} a \\
& \text { Success }_{N D} a \ggg f=f a \\
& \text { Fail }_{N D} \ggg f=\text { Fail }_{N D} \\
& O r_{N D} l r \quad \ggg f=O r_{N D}(l \ggg f)(r \gg f)
\end{aligned}
$$

${ }^{3}$ In Haskell we work in the domain $\langle\mathcal{P}(\mathbb{N} \times \mathbb{N}) \cup\{\perp\}$, $\rangle$, where every type is inhabited by $\perp$, representing non-termination, and $\perp \sqsubseteq v$ for any value $v$. 
This monad instance substitutes $f$ a for every Success $_{N D} a$ in the data structure, leaves Fail untouched, and recurses on both branches of $O r l r$. With this monad instance, the example (1 ? $2)+(1 ? 2)$ is expressed as:

$$
\begin{gathered}
\text { exampleND::ND } \text { Int } \\
\text { exampleND= do } x \leftarrow \text { return } 1 \text { 'Or }{ }_{N D} \text { ' return } 2 \\
y \leftarrow \text { return } 1 \text { 'Or } r_{N D} \text { ' return } 2 \\
\text { return }(x+y)
\end{gathered}
$$

Values of type $N D a$ are abstract syntax trees. The function exampleND constructs such an abstract syntax tree. The interpreter $n d$ decodes this tree according to the semantics defined by $\llbracket \cdot \rrbracket$. It turns Success into a singleton set, Fail into an empty set, and $\mathrm{Or}$ into the union of the interpretations of its branches.

$$
\begin{aligned}
& \text { nd ::Ord } a \Rightarrow N D a \rightarrow \text { Set } a \\
& \text { nd }\left(\text { Success }_{N D} a\right)=\text { singleton } a \\
& \text { nd } \text { Fail }_{N D}=\text { empty } \\
& \text { nd }\left(\text { Or }_{N D} l r\right)=\text { union }(\text { nd } l)(\text { nd } r) \\
& \text { やnd exampleND } \\
& \text { fromList }[2,3,4]
\end{aligned}
$$

The equivalent of pair in the abstract syntax is pair $N D$ :

$$
\begin{aligned}
& \text { pairND :: ND (Int, Int }) \\
& \text { pairND }=\text { return }(1,2) \text { 'Or }{ }_{N} \text { ' fmap swap pairND where } \\
& \quad \text { swap }(x, y)=(y, x) \\
& \text { \nd pairND } \\
& \text { fromList ... }
\end{aligned}
$$

Encoding the non-deterministic syntax as a data type does not solve the problem of non-termination. This can be explained by looking at the syntax tree for pairND in Figure 1: it is an infinite tree with an $\mathrm{Or}$ node at the root, a Success node in the left branch, and in the right branch another tree with $O r$ at the root after which the pattern repeats. Obviously the interpreter $n d$ does not terminate when interpreting such an infinite tree.

We have little chance of processing the infinite tree in a finite time if we do not represent it in a finite way. Yet the current recursive form of pair ND hides the recursive call in the function body, making the construction of an infinite tree unavoidable.

\section{EXPLICATING RECURSION}

In order to obtain a finite syntax tree, we have to once more change the representation of non-deterministic computations. First, we add a constructor to the abstract syntax to explicitly represent a recursive call. Second, we replace all recursive calls with this constructor, and finally, we define a new effect handler that interprets the now finite syntax tree, producing the desired solution. The following data type models recursive calls in addition to non-determinism:

$$
\begin{aligned}
& \text { data NDRec } i \text { o } \\
& =\text { Success } a \\
& \text { Fail } \\
& -(1) \\
& \text { Or (NDRec } i \text { o } a)(N D R e c i o a)-(2) \\
& \text { Rec } i(o \rightarrow N D R e c i o a)
\end{aligned}
$$

The first three constructors (1-3) capture non-determinism, exactly like the previously defined data type $N D$. The last constructor (4) captures a recursive call: $\operatorname{Rec} a k$ represents a recursive call with argument $a:: i$, and continuation $k:: o \rightarrow N D R e c$ i $o$ a. For convenience, we define four additional smart constructors. The smart constructor rec performs a recursive call and immediately wraps the result in a successful computation:

$$
\begin{aligned}
& \text { rec }:: i \rightarrow \text { NDRec } i \text { o o } \\
& \text { rec } i=\text { Rec } i \text { Success }
\end{aligned}
$$

The smart constructor choice picks a computation from a list in a non-deterministic fashion.

$$
\begin{aligned}
& \text { choice }::[\text { NDRec } i \text { o a }] \\
& \text { choice }=\text { foldr Or Fail }
\end{aligned}
$$

The smart constructor choose picks an element from a list in a nondeterministic fashion.

$$
\begin{aligned}
& \text { choose }::[a] \rightarrow \text { NDRec } i \text { o a } \\
& \text { choose }=\text { choice } \circ \text { map Success }
\end{aligned}
$$

The smart constructor guard returns () if its argument is true and fails otherwise.

$$
\begin{aligned}
& \text { guard }:: \text { Bool } \rightarrow \text { NDRec } i \text { o () } \\
& \text { guard } b=\text { if } b \text { then return () else Fail }
\end{aligned}
$$

The data type NDRec is again a free monad, the corresponding monad instance is:

$$
\begin{array}{ll}
\text { instance Monad }(\text { NDRec } i \text { o) where } & -(1) \\
\text { return } a=\text { Success } a & -(2) \\
\text { Success } a \ggg f=f a & -(3) \\
\text { Fail } \ggg f=\text { Fail } & -(4) \\
\text { Or } l r \ggg f=\operatorname{Or}(l \ggg f)(r \gg f) & -(5) \\
\text { Rec } i k \ggg f=\operatorname{Rec} i(\lambda x \rightarrow k x \gg f) & -(6)
\end{array}
$$

Lines 1-5 are identical to the Monad instance for ND. Line (6) defines that $\ggg$ of a recursive call is obtained by a new recursive call to the same argument, but with an extended continuation. ${ }^{4}$

As an example, consider this latest incarnation of pair, written in the NDRec syntax:

$$
\begin{array}{r}
\text { pairNDRec }::() \rightarrow \text { NDRec }()(\text { Int }, \text { Int })(\text { Int }, \text { Int }) \\
\text { pairNDRec }()=\text { return }(1,2) \text { 'Or' do }(x, y) \leftarrow \text { rec }() \\
\text { return }(y, x)
\end{array}
$$

We interpret this program in the next section.

\section{EFFECT HANDLER FOR EXPLICIT RE- CURSION}

\subsection{Denotational semantics}

In this section we formalize the meaning of the abstract syntax. The meaning of a non-deterministic function $f$ of type $I \rightarrow$ NDRec $I O O$, mapping $I$ onto NDRec $I O O$ is given by a function $\llbracket f \rrbracket: I \rightarrow \mathcal{P}(O)$. This function maps values of type $I$ onto a subset of the values described by the type $O$.

$$
\llbracket \cdot \rrbracket:(I \rightarrow N D R e c I O O) \rightarrow(I \rightarrow \mathcal{P}(O))
$$

Let us first define the semantics of an easier case: suppose we already have an environment $s: I \rightarrow \mathcal{P}(O)$ that contains a partial set of solutions for every call $f(a)$, i.e.

$$
s(a) \subseteq \llbracket f \rrbracket(a) \text { for every } a \in I
$$

\footnotetext{
${ }^{4}$ The new continuation is the Kleisli composition $k \Longleftrightarrow f$ where $(\Longrightarrow)::$ Monad $m \Rightarrow(a \rightarrow m b) \rightarrow(b \rightarrow m c) \rightarrow(a \rightarrow m c)$. ${ }^{5}$ In deference to mathematical convention, we use uppercase characters for meta-variables $I$ and $O$ when using mathematical syntax.
} 
Then for every syntax tree $t: N D I O O$ we can define a semantic function $\mathcal{R} \llbracket t \rrbracket(s)$. This semantic function gives us the set of results associated with $t$, given $s$. Because the $N D I O O$ data type is inductive, we define the function $\mathcal{R} \llbracket \cdot \rrbracket$ using structural recursion, as follows:

$$
\begin{array}{lll}
\mathcal{R} \llbracket \cdot \text { NDRec } I & O & O \rightarrow(I \rightarrow \mathcal{P}(O)) \rightarrow \mathcal{P}(O) \\
\mathcal{R} \llbracket \text { Success } x & \rrbracket(s)=\{x\} \\
\mathcal{R} \llbracket \text { Fail } & \rrbracket(s)=\emptyset \\
\mathcal{R} \llbracket \text { Or } l r & \rrbracket(s)=\mathcal{R} \llbracket l \rrbracket(s) \cup \mathcal{R} \llbracket r \rrbracket(s) \\
\mathcal{R} \llbracket \text { Rec } i k & \rrbracket(s)=\bigcup_{x \in s(i)} \mathcal{R} \llbracket k(x) \rrbracket(s)
\end{array}
$$

The two base cases are fairly simple: in the deterministic case (2) the result is just a singleton set of the result, and in the failure case (3) it is the empty set.

There are two inductive cases as well: a binary choice (4) and a recursive call (5). A binary choice is handled by taking the union of the results of the left and right branches. A recursive call has an argument $i$ and a continuation $k$. The result is obtained by finding the set of outcomes in the environment $s$, and then applying the continuation $k$ to every element $x$ in $s(i)$, and taking the union of the result.

Now we can of define $\llbracket \cdot \rrbracket$ as follows: since $\mathcal{R} \llbracket f(a) \rrbracket(\llbracket f \rrbracket)$ gives the set of outcomes of $f(a)$ given environment $\llbracket f \rrbracket$, and $\llbracket f \rrbracket(a)$ gives the set of outcomes of $f(a)$, the following must hold about $\mathcal{R} \llbracket f(a) \rrbracket(\llbracket f \rrbracket)$ :

$$
\begin{aligned}
& \mathcal{R} \llbracket f(a) \rrbracket(\llbracket f \rrbracket)=\llbracket f \rrbracket(a) \quad(\forall a \in I) \\
& \text { [ equivalence of } \lambda \text {-abstraction and } \forall \text {-quantification }] \\
\Longleftrightarrow & \lambda a \cdot \mathcal{R} \llbracket f(a) \rrbracket(s)=\lambda a \cdot \llbracket f \rrbracket(a) \\
\Longleftrightarrow & \lambda a \cdot \mathcal{R} \llbracket f(a) \rrbracket(\llbracket f \rrbracket)=\llbracket f \rrbracket[\eta \text {-reduction }] \\
\Longleftrightarrow & \llbracket f \rrbracket \text { is a fixed point of } \lambda s \cdot \lambda a \cdot \mathcal{R} \llbracket f(a) \rrbracket(s)
\end{aligned}
$$

Now all that remains is to choose a canonical fixed point for $\llbracket f \rrbracket$, such that it corresponds to the desired meaning. Note that environments of the type $I \rightarrow \mathcal{P}(O)$ (such as $\llbracket f \rrbracket$ ) are ordered by the ordering relation $\sqsubseteq$ :

$$
f \sqsubseteq g \Longleftrightarrow \forall a \in I: f(a) \subseteq g(a)
$$

The desired fixed point is the least fixed point ${ }^{6}$ denoted by $l f p(\cdot)$, when fixed points are ordered by $\sqsubseteq$ :

$$
\llbracket f \rrbracket=l f p(\lambda s \cdot \lambda a \cdot \mathcal{R} \llbracket f(a) \rrbracket(s))
$$

To make this more concrete, consider the denotational semantics of pairNDRec.

$$
\begin{aligned}
& \llbracket \operatorname{pairNDRec}() \rrbracket(()) \\
& =l f p(\lambda s \cdot \lambda() \cdot \mathcal{R} \llbracket \text { pairNDRec }() \rrbracket(s))(()) \quad \text { [ by }(6)] \\
& {[\lambda() \cdot\{(1,2),(2,1)\} \text { is the least fixed point }]} \\
& =(\lambda() \cdot\{(1,2),(2,1\}()) \\
& {[\text { function application ] }} \\
& \{(1,2),(2,1)\}
\end{aligned}
$$

\footnotetext{
${ }^{6}$ The least fixed point is well defined, as the function is always continuous (if the syntax tree is finite) and therefore monotonic, but the domain $I \rightarrow \mathcal{P}(O)$ may not possess the finite ascending chain property, preventing us from computing the solution in a finite amount of time.
}

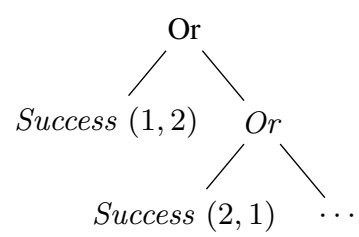

Figure 1: Infinite syntax tree created by pair $N D$.

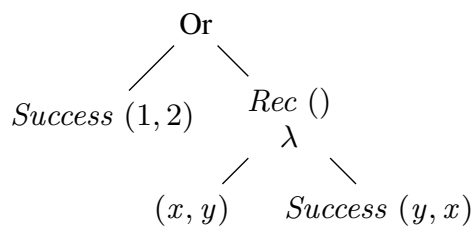

Figure 2: Finite syntax tree created by pairNDRec ().

We can see that $\lambda() .\{(1,2),(2,1)\}$ is the least fixed point because it is a fixed point and it is contained in every other fixed point.

\subsection{Effect Handler Implementation}

In this section we provide a Haskell implementation that correspond to the denotational semantics from the previous section. This implementation comes in the form of an effect handler [10, 27]. Like the denotational semantics, the effect handler is split into two parts: a part that delivers the solution for the entire function by computing a least fixed point (similar to $\llbracket \cdot \rrbracket$ ), and the counterpart of $\mathcal{R} \llbracket \cdot \rrbracket$, which, given an environment, computes the results for one syntax tree.

The first part is called runNDRec and the second part is called go. The effect handler first computes the least fixed point of the step function. The step function is a function that takes an environment (of type Map $i$ (Set o) from Data. Map ${ }^{7}$ ) and obtains a new environment by running $g o$ for every entry in the environment. The fixed point of this function is again an environment, in which we look up $i_{0}$, the second argument to runNDRec.

$$
\begin{aligned}
& \text { runNDRec }::(\text { Ord } i \text {, Ord o }) \\
& \quad \Rightarrow(i \rightarrow \text { NDRec } i \text { o o }) \rightarrow i \rightarrow \text { Set o } \\
& \text { runNDRec expr } i_{0}=\text { lfp step } s_{0} ! i_{0} \text { where } \\
& \quad s_{0}=M . \operatorname{singleton} i_{0} \text { empty } \\
& \quad \text { step } m=\text { foldr }(\lambda k \rightarrow \text { go } k(\text { expr } k)) m(\text { M.keys } m)
\end{aligned}
$$

The function $g o$, like $\mathcal{R} \llbracket \cdot \rrbracket$ proceeds by case analysis on the syntax tree. However, unlike $\mathcal{R} \llbracket \cdot \rrbracket$, go updates an environment instead of just returning a set of solutions. This difference is mainly for programming convenience.

$$
\begin{aligned}
& \text { go :: (Ord } i \text {, Ord o) } \\
& \Rightarrow i \rightarrow N D R e c \text { i o o } \\
& \rightarrow \text { M.Map } i(\text { Set o }) \rightarrow \text { M.Map } i(\text { Set o }) \\
& \text { go } i \text { (Success a) } m=M \text {.insertWith union } i \text { (singleton } a) m \\
& \text { go } i \text { Fail } \quad m=m \\
& \text { go } i(\text { Or } l r) \quad m=\text { go } i r(\text { go } i l m) \\
& \text { go } i(\operatorname{Rec} j k) \quad m=\text { case M.lookup } j m \text { of } \\
& \text { Nothing } \rightarrow \text { M.insert } j \text { empty } m \\
& \text { Just } s \rightarrow \text { foldr (go } i \circ k) m \text { (toList } s)
\end{aligned}
$$

${ }^{7}$ Functions and types residing in Data. Map are imported with the qualifier M, except the lookup operator “!”. 




Figure 3: The graph used in the reachable and shortest path example.

If the syntax tree contains a deterministic result (line 4), we simply add this result to the environment. If the syntax contains a failure (line 5), the environment remains unchanged. When presented with a binary choice (line 6), we first update the environment according to the left branch and then according to the right branch. Finally, in the case of a recursive call Rec $j k$, we first check if the map contains an entry for the argument $j$ (line 7). If it has no such entry, we add an entry containing the empty set (line 8), otherwise we update the environment based on the existing elements (line 9). Inserting an empty entry for $j$, ensures that the next iteration of step also updates the map for expr $j$.

We still need a function $l f p$ to iteratively compute a least fixed point. Given an initial value, this function keeps calling itself until the result no longer changes.

lfp :: Eq $a \Rightarrow(a \rightarrow a) \rightarrow a \rightarrow a$

lfp $f a_{0}=$ let $a_{1}=f a_{0}$ in if $a_{1} \equiv a_{0}$ then $a_{1}$ else lfp $f a_{1}$

As an example, consider the semantics of pairNDRec:

や runNDRec pairNDRec ()

fromList $[(1,2),(2,1)]$

The denotational semantics and its Haskell implementation coincide as expected, but only up to termination (as we show in Section 6).

Graph Example We compute reachability in a cyclic directed graph using our non-determinism framework. We use the adjacency list representation of a graph where every Node has a label (of type String) and a list of adjacent nodes:

data Node $=$ Node $\{$ label :: String, adj :: $[$ Node $]\}$

instance $E q \quad$ Node where $n_{1} \equiv n_{2}=$ label $n_{1} \equiv$ label $n_{2}$

instance Ord Node where $n_{1} \leqslant n_{2}=$ label $n_{1} \leqslant$ label $n_{2}$

Our example graph (see Figure 3) consists of five nodes. It contains two cycles: from 3 to 4 and back, and from 5 to itself.

$$
\begin{aligned}
{\left[n_{1}, n_{2}, n_{3}, n_{4}, n_{5}\right]=} & {\left[\text { Node "1" }\left[n_{2}, n_{5}\right],\right.} \\
& \text { Node "2" }\left[n_{3}\right], \\
& \text { Node "3" }\left[n_{4}\right], \\
& \text { Node "4" }\left[n_{3}, n_{1}\right], \\
& \text { Node "5" } \left.\left[n_{5}\right]\right]
\end{aligned}
$$

Reachability is straightforwardly computed as:

reach $::$ Node $\rightarrow$ NDRec Node Node Node

reach $n=$ return $n^{\prime}$ Or' $^{\text {' }}$ choose $(\operatorname{adj} n) \gg$ rec $)$

i.e. $n$ is reachable from $n$ and all nodes reachable from a neighbor of $n$ are reachable from $n$.

》 runNDRec reach $n_{1}$

fromList [Node 1, Node 2, Node 3, Node 4, Node 5]

\begin{tabular}{lll}
\hline $2: \emptyset,[]$ & \\
\hline $2: \emptyset,[]$ & $1: \emptyset,\left[\right.$ go $\left.2 \circ k_{0}\right]$ \\
\hline $2: \emptyset,[]$ & $1:\{1\},\left[\right.$ go $\left.2 \circ k_{0}\right]$ \\
\hline $2: \emptyset,[]$ & $1:\{1\},\left[\right.$ go $\left.2 \circ k_{0}\right]$ & $0: \emptyset,\left[\right.$ go $\left.1 \circ l_{1}\right]$ \\
\hline $2: \emptyset,[]$ & $1:\{1\},\left[\right.$ go $\left.2 \circ k_{0}\right]$ & $0:\{0\},\left[\right.$ go $\left.1 \circ l_{1}\right]$ \\
\hline $2:\{2\},[]$ & $1:\{1\},\left[\right.$ go $\left.2 \circ k_{0}\right]$ & $0:\{0\},\left[\right.$ go $\left.1 \circ l_{1}\right]$ \\
\hline $2:\{2\},[]$ & $1:\{1\},\left[\right.$ go $\left.2 \circ k_{0}\right]$ & $0:\{0\},\left[\right.$ go $\left.1 \circ l_{1}\right]$ \\
\hline
\end{tabular}

Table 1: The trace of the environment for runNDk fib 2 .

\section{DEPENDENCY TRACKING}

The effect handler that was provided in Section 4 computes the least fixed point in a very naive way, resulting in sub par performance. To illustrate the problem, consider the following program to compute the Fibonacci numbers:

$$
\begin{aligned}
& \text { fib : Int } \rightarrow \text { NDRec Int Int Int } \\
& \text { fib } n \mid n \equiv 0=\text { return } 0 \\
& \mid n \equiv 1=\text { return } 1 \\
& \mid \text { otherwise }=\operatorname{do~} f_{1} \leftarrow \operatorname{rec}(n-1) \\
& \quad \quad f_{2} \leftarrow \operatorname{rec}(n-2) \\
& \quad \text { return }\left(f_{1}+f_{2}\right)
\end{aligned}
$$

The evolution of the environment for runNDRec fib 2 after each application of step is shown in Table 1 (ignore the list after the comma for now). Every value in the environment is recomputed in every step. The work performed by the effect handler is monotonic: the work performed by a single iteration is also performed in all later iterations. In the case of $f i b$ this leads to an $\mathcal{O}\left(n^{2}\right)$ runtime. $^{8}$

This duplication of work has two sources:

1. The naive least fixed point computation lfp iteratively applies the step function which folds over all keys in the environment, even when the set of outcomes of that key has not changed.

2. The function go computes too much: the only part of the syntax tree that is influenced by a recursive call Rec $j k$ is the continuation $k$ of that recursive call. Therefore only the continuation needs to be recomputed.

Although efficiency is not the main focus of this paper, we would be remiss not to address such a glaring problem. Especially since the solution is simple: both problems can be solved by keeping track of which continuation depends on which recursive call (identified by its argument), and only evaluating the continuation once for every new value of the recursive call.

Figure 4 shows the code for the interpreter runNDk that tracks dependencies. This effect handler relies on seven auxiliary functions defined for the type Env $i$ o. This type abstracts over environments that contain continuations in addition to a set of values. We show their signatures and implementations in Figure 4.

The computation starts with the call to go on line 1, which returns the environment after the least fixed point computed. From this environment the result is extracted using !!!. The go function proceeds by case analysis: if the syntax tree is Success $x$ and the value $x$ is not yet in the environment, the environment is updated by sequentially applying every continuation that is currently in the environment (line 2-3). If the syntax tree is a failed computation the environment is unchanged (line 4). If the syntax tree is a binary choice (line 5) the environment is updated first for the left

\footnotetext{
${ }^{8}$ modulo a logarithmic factor for the use of finite maps.
} 
branch, then for the right branch. If the syntax tree is a recursive call Rec $j k$ (line 6-10), and there is no entry for $j$ in the map (line 9), i.e. this is the first time we see a call to $j$, a continuation for $j$ is added (line 7) and the recursive function is evaluated for $j$. Otherwise (line 10), a new continuation is added for $j$, and this continuation is applied to every known result of $j$. The least fixed point is reached when the ifNew check on line 2 no longer succeeds.

The trace for runNDk fib 2 is identical to the trace for runND fib 2, but now only a single entry is updated in each iteration. Moreover, the result from the call to 1 only triggers the continuation $k_{0}=\lambda f_{1} \rightarrow \operatorname{Rec} 0\left(\lambda f_{2} \rightarrow\right.$ return $\left.\left(f_{1}+f_{2}\right)\right)$ and the result of 0 only triggers computation of $l_{1}=\lambda f_{2} \rightarrow$ return $\left(1+f_{2}\right)$, instead of the entire syntax tree $f i b 2$.

In summary, we obtain a linear runtime for $f i b$.

\section{LATTICES}

Reconsider the graph defined in Section 4. Instead of just finding all reachable nodes, we may want to additionally know the length of the shortest path. The program $s p$ is a first attempt at computing the shortest path between src and dst.

$$
\begin{aligned}
& \text { sp :: Node } \rightarrow \text { Node } \rightarrow \text { NDRec Node Int Int } \\
& \text { sp dst src } \\
& \quad \mid \text { dst } \equiv \text { src }=\text { return } 0 \\
& \mid \text { otherwise }=\text { choose }(\text { adj src }) \gg \text { rec } \gg \text { return } \circ(+1)
\end{aligned}
$$

The denotational semantics gives us the following value for the expression $s p n_{1}$ rec $n_{2}$ :

$$
\llbracket s p n_{1} \operatorname{rec} \rrbracket\left(n_{2}\right)=\{2,4,6,8, \ldots\}
$$

Since this value is infinite, the effect handler does not terminate. In order to implement a terminating effect handler, we need to first define an alternative denotational semantics that at least produces a finite value, which we can then compute in a finite amount of time. Lattice-based Semantics Instead of powersets $\mathcal{P}(O)$, we generalize the semantic domain to an arbitrary complete lattice $L$. On this domain we define a new denotational semantics for non-deterministic computations. The signature of this denotational semantics is:

$$
\llbracket \cdot \rrbracket_{L}:(I \rightarrow N D R e c I L L) \rightarrow(I \rightarrow L)
$$

where $\langle L, \sqsubseteq\rangle$ is a complete lattice.

A complete lattice is a partially ordered set $\langle S, \sqsubseteq\rangle$ such that for every subset $X \in S$ there exists a least upper bound, i.e. an element $\sqcup X$ that is the least element that is larger than every element in $X$, more formally:

DEFINITION 1 (COMPLETE LATTICE). A complete lattice $\langle L, \sqcup\rangle$ is a partially ordered set $\langle L, \sqsubseteq\rangle$ where $\forall X \subseteq L$ there exists a least upper bound $\sqcup X \in L$, such that:

$$
\forall z \in L: \sqcup X \sqsubseteq z \Longleftrightarrow \forall x \in X: x \sqsubseteq z
$$

It follows from the definition that $\sqcup X$ is unique. A complete lattice $L$ always has a least element $\perp$ that is smaller than all other elements. It corresponds to the least upper bound of the empty set: $\perp=\sqcup \emptyset$. With a slight abuse of notation we also write $a \sqcup b$ to denote the least upper bound $\sqcup\{a, b\}$, pronounced "join".

As before, we first consider the semantics for the case where we already possess an environment $s: I \rightarrow L$ containing the (partial) solution for every call $f(a)$, i.e.

$$
s(a) \sqsubseteq \llbracket f \rrbracket_{L}(a) \text { for every } a \in I
$$

Then for every syntax tree $t: N D R e c I L L$ we can define the denotational semantics $\mathcal{R} \llbracket \cdot \rrbracket_{L}$ in the new setting:

$$
\begin{array}{ll}
\mathcal{R} \llbracket \cdot \rrbracket_{L}: \text { NDRec } I L L \rightarrow(I \rightarrow L) \rightarrow L \\
\mathcal{R} \llbracket \text { Success } x & \rrbracket_{L}(s)=x \\
\mathcal{R} \llbracket \text { Fail } & \rrbracket_{L}(s)=\perp \\
\mathcal{R} \llbracket \text { Or } l r & \rrbracket_{L}(s)=\mathcal{R} \llbracket l \rrbracket_{L}(s) \sqcup \mathcal{R} \llbracket r \rrbracket_{L}(s) \\
\mathcal{R} \llbracket \text { Rec } j k & \rrbracket_{L}(s)=\mathcal{R} \llbracket k(s(j)) \rrbracket_{L}(s)
\end{array}
$$

This semantics is very similar to the previously defined semantics for sets. In particular, the semantics $\mathcal{R} \llbracket \cdot \rrbracket$ almost corresponds to the semantics $\mathcal{R} \llbracket \cdot \rrbracket_{L}$ specialized to the powerset of the output type $\left(\mathcal{R} \llbracket \cdot \rrbracket_{\mathcal{P}(O)}\right)$. We will make this relationship more precise in Section 6.1.

For $\llbracket f \rrbracket_{L}$ we would again like to compute the least fixed point of $\lambda s . \lambda a .\left(\mathcal{R} \llbracket f(a) \rrbracket_{L}(s)\right)$. However, unlike for sets, this function is not guaranteed to be monotonic for arbitrary lattices in general, so a least fixed point might not always exist. Instead, we define the operator $\Uparrow$ :

DEFINITION 2. The operator $\Uparrow$ is defined as:

$$
\begin{aligned}
f \Uparrow 0 & =\perp \\
f \Uparrow(i+1) & =f \Uparrow i \sqcup f(f \Uparrow i) \\
f \Uparrow \infty & =\bigsqcup_{i=0}^{\infty}(f \Uparrow i)
\end{aligned}
$$

Observe that the sequence $f \Uparrow 0, f \Uparrow 1, f \Uparrow 2, \ldots, f \Uparrow \infty$ is non-decreasing. ${ }^{9}$

If the domain of $f$ has the finite ascending chain property this sequence is finite, and then $f \Uparrow \infty$ must be a fixed point. Thus, we define $\llbracket \cdot \rrbracket_{L}$ as:

$$
\llbracket f \rrbracket_{L}=\left(\lambda s \cdot \lambda a \cdot \mathcal{R} \llbracket f(a) \rrbracket_{L}(s)\right) \Uparrow \infty
$$

Moreover, if $\lambda s . \lambda a . \mathcal{R} \llbracket f(a) \rrbracket_{L}(s)$ is continuous, then $\Uparrow$ computes the least fixed point, i.e.

$l f p\left(\lambda s . \lambda a \cdot \mathcal{R} \llbracket f(a) \rrbracket_{L}(s)\right)=\left(\lambda s \cdot \lambda a \cdot \mathcal{R} \llbracket f(a) \rrbracket_{L}(s)\right) \Uparrow \infty=\llbracket f \rrbracket_{L}$

Otherwise, $\Uparrow$ may compute a fixed point that is strictly greater than the least fixed point. In practice, however, almost all functions are continuous.

Shortest Path Example For our shortest path problem, the relevant partial order is $\langle\mathbb{N} \cup\{\infty\}$, $\rangle$ where

$$
a \sqsubseteq b \Longleftrightarrow a=\infty \text { or } b \leqslant a
$$

which is the reverse order of the canonical order on $\mathbb{N}$, i.e. smaller (shorter) is better. Since the canonical order is well-founded (i.e. has no infinite descending chains), $\sqsubseteq$ has the finite ascending chain property. The relevant lattice is $\langle\mathbb{N} \cup\{\infty\}, \sqcup\rangle$ where

$$
\sqcup X= \begin{cases}\infty & \text { if } X=\emptyset \\ \min _{\sqsubseteq} X & \text { otherwise }\end{cases}
$$

In this lattice the least fixed point exists, since continuation returno $(+1)$ in $s p n_{1}$ is continuous.

$$
\llbracket s p n_{1} \rrbracket_{\mathbb{N} \cup\{\infty\}}\left(n_{2}\right)=3
$$

${ }^{9}$ For environments, assume the order $\preccurlyeq$ defined as:

$$
s_{1} \preccurlyeq s_{2} \Longleftrightarrow \forall a \in I: s_{1}(a) \sqsubseteq s_{2}(a)
$$




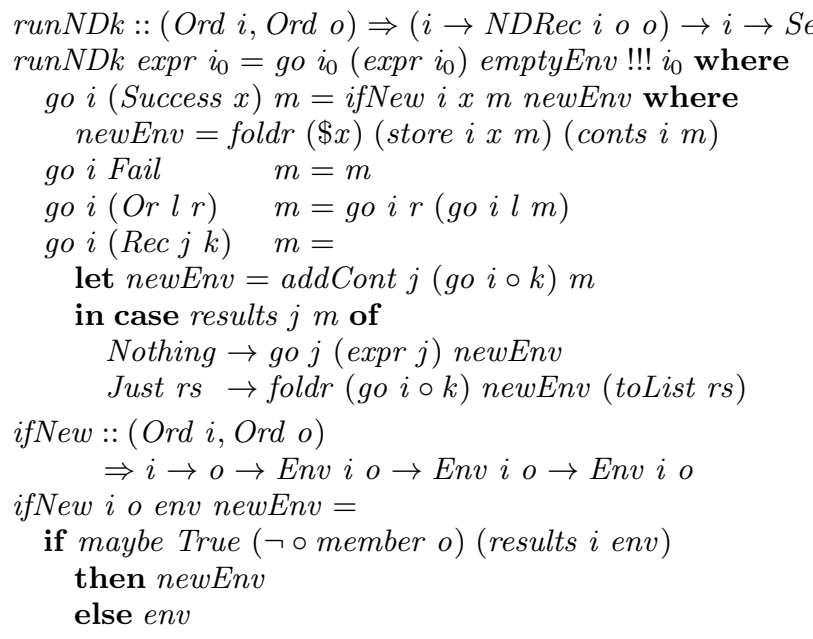

type Env $i$ o $=$ M.Map $i\left(\right.$ Set $\left.o,\left[\begin{array}{lll}C & i & o\end{array}\right]\right)$

emptyEnv :: Env $i$ o

emptyEnv $=$ M.empty

store $:($ Ord $i$, Ord $o) \Rightarrow i \rightarrow o \rightarrow$ Env $i o \rightarrow$ Env $i o$ store $i o=M$.insert With mappend $i$ (singleton $o,[]$ ) results :: Ord $i \Rightarrow i \rightarrow$ Env $i$ o $\rightarrow$ Maybe (Set o) results $i=$ fmap fst $\circ$ M.lookup $i$

(!!!) :: Ord $i \Rightarrow$ Env $i$ o $\rightarrow i \rightarrow$ Set o

$m ! ! ! ~ i=$ fromJust (results $i \mathrm{~m}$ )

conts $::$ Ord $i \Rightarrow i \rightarrow$ Env $i o \rightarrow[o \rightarrow$ Env $i o \rightarrow$ Env $i o]$ conts $i=$ map runC $\circ M$. findWithDefault []$i \circ$ fmap snd

addCont :: (Ord $i$, Ord o $) \Rightarrow i \rightarrow(o \rightarrow$ Env $i o \rightarrow$ Env $i$ o $)$ $\rightarrow$ Env $i$ o $\rightarrow$ Env $i o$

addCont $i k=M$.insertWith mappend $i$ (empty, $\left.\left[\begin{array}{ll}C & k\end{array}\right]\right)$

Figure 4: Effect Handler and Environment type for dependency tracking.

The length of the shortest path from node 2 to node 1 is indeed 3 . When a path does not exist, we get the following length:

$$
\llbracket s p n_{1} \rrbracket_{\mathbb{N} \cup\{\infty\}}\left(n_{5}\right)=\infty
$$

Notice the similarity to solving a problem with Dynamic Programming. The idea of dynamic programming is to compute the optimal solution to a problem by combining optimal solutions to smaller instances of the same problem. In this setting, recursive calls correspond to instances of the same problem. The lattice allows us to keep only the optimal solution to a given instance, from which the result of a larger instance is computed.

Subset Sum Example Another example is the following:

Given a list of integer numbers and an integer s, find the shortest non-empty sublist that sums to $s$.

To solve this problem, first observe that lists form a lattice when ordered by descending length, if we adjoin a bottom element InfList representing any list of infinite length. This is embodied by the Shortest-datatype (see Section 4.2 for the definition of Lattice).



The function sss returns the Shortest list which sums to its first argument and is a sublist of of its second argument.

$$
\begin{aligned}
& \text { sss }::(\text { Int },[\text { Int }]) \rightarrow \text { NDRec }(\text { Int },[\text { Int }]) \text { Shortest Shortest } \\
& \text { sss }(n,[]) \\
& \mid n \equiv 0=\text { return }(\text { List }[]) \\
& \mid \begin{array}{l}
\text { otherwise }=\text { Fail } \\
\text { sss }(n, x: x s)=\text { choice }[\operatorname{rec}(n, x s), \\
\text { fmap }(\text { cons } x)(\operatorname{rec}(n-x, x s))]
\end{array}
\end{aligned}
$$

When the input list is empty we can only construct an empty list with sum 0 . When the input list is non-empty, the shortest list sum- ming to $n$ is obtained by either recursively searching for a list summing to $n$, or cons-ing $x$ onto a list that sums to $n-x$.

The function $\mathcal{R} \llbracket s s s(n, x s) \rrbracket_{L}$ is continuous for all $(n, x s)$. Moreover, the environment possesses a finite ascending chain property: every recursive call decreases the size of the input list by one. Then, given a finite input list, there can only be a finitely many recursive calls.

For instance, consider the application of $s s s$ to $(10,[5,0,5])$ :

$$
\llbracket s s s \rrbracket(10,[5,0,5])=\operatorname{List}[5,5]
$$

Indeed sum $[5,5] \equiv 10$. Note that the list $[5,0,5]$ itself also sums to 10 . However, only the shortest list is retained.

Grammar Analysis example We demonstrate the added power of the lattice-based semantics by solving a simple grammar analysis problem. Informally, a grammar is a list of rules or productions:

$$
\text { type Grammar }=[\text { Production }]
$$

A production is of the form

$$
\text { data Production }=\text { Symbol } \longmapsto[\text { Symbol }]
$$

There is a single Symbol to the left of $\longmapsto$, called the head, and zero or more symbols, the body, on the right hand side. Symbols can be either terminals or non-terminals:

$$
\begin{aligned}
& \text { type Symbol }=\text { Char } \\
& \text { isTerminal }:: \text { Symbol } \rightarrow \text { Bool } \\
& \text { isTerminal } s=\neg(\text { isUpper } s)
\end{aligned}
$$

Terminals may not appear in the head of a rule. For our purpose we distinguish terminals and non-terminals based on whether they are upper or lower case characters.

Now, consider the following grammar:

$$
\begin{aligned}
& \text { grammar :: Grammar } \\
& \text { grammar }=\left[{ }^{\prime} \mathrm{E}^{\prime} \longmapsto \mathrm{T} \mathrm{Z} ", \quad \mathrm{E}^{\prime} \longmapsto "(\mathrm{E}) \mathrm{"}\right. \text {, } \\
& \mathrm{Z}^{\prime} \longmapsto \mathrm{Z}^{\prime} \mathrm{T} \mathrm{Z}^{\prime}, \mathrm{Z}^{\prime} \longmapsto \mathrm{Z}^{\prime}+(\mathrm{E}) \text { ", } \\
& \text { ' } \mathrm{Z}^{\prime} \longmapsto " \text { ", } \\
& { }^{\prime} \mathrm{T}^{\prime} \longmapsto \mathrm{a} ", \quad \mathrm{~T}^{\prime} \longmapsto \mathrm{M} \text { "] }
\end{aligned}
$$

This grammar describes a simple expression language consisting of one identifier $(a)$, one literal (1) and a binary operator $(+)$. We implement a program that analyses grammars for nullability: a symbol is Nullable if it derives the empty string. Nullability is charac- 
terized by a function from Symbol to $A n y$, where $A n y$ is a lattice of booleans, where join is disjunction and False is bottom.

$$
\begin{aligned}
& \text { newtype Any }=\text { Any }\{\text { getAny }:: \text { Bool }\} \\
& \text { instance Lattice Any where } \\
& \quad \text { bottom }=\text { Any False } \\
& \text { join }(\text { Any } a)(\text { Any } b)=\text { Any }(a \vee b)
\end{aligned}
$$

Given this lattice, the implementation of nullable is straightforward:

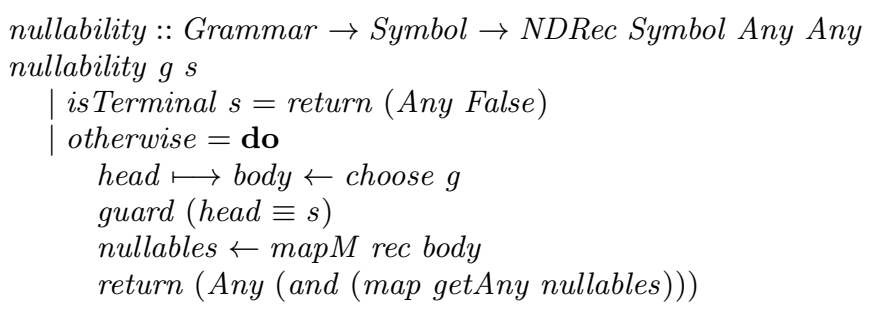

A terminal symbol is never nullable. In order to decide if a nonterminal symbol $s$ is nullable, we need to check if there exists a production with $s$ in the head and with a body consisting of nullable symbols. Note that $s$ may also occur in the body.

We evaluate nullability for grammar:

$$
\begin{aligned}
& \llbracket \text { nullability grammar } \rrbracket_{A n y}\left({ }^{\prime} \mathrm{T} '\right)=\text { Any False } \\
& \llbracket \text { nullability grammar } \rrbracket_{A n y}\left({ }^{\prime} \mathrm{Z}^{\prime}\right)=\text { Any True } \\
& \llbracket \text { nullability grammar } \rrbracket_{A n y}\left({ }^{\prime} \mathrm{E}^{\prime}\right)=\text { Any False }
\end{aligned}
$$

We have demonstrated that a semantics based on lattices allows us to tackle problems we could not previously solve in a finite amount of time. Note that we again arrived at this solution by computing a least fixed point, but of a different function. Furthermore, only the semantics of the syntax has changed, the syntax itself is unmodified. Hence, to implement this semantics only the effect handler needs to be reworked.

\subsection{Generalized Effect Handler}

This section shows that the semantics from Section 6 is strictly more general than the semantics from Section 4.2. The NDRec monad is a free monad. More precisely, it is the coproduct monad of two other free monads respectively implementing non-determinism and recursion. In this section we make this coproduct explicit by following the data types à la carte approach [20]. All free monads have the following shape:

$$
\text { data Free } f a=\text { Return } a \mid \text { Free }(f \text { (Free } f a))
$$

They either return a pure value (Return) or an impure effect (Free), constructed using $f$. When $f$ is a Functor, Free $f$ is a monad:

$$
\begin{aligned}
& \text { instance Functor } f \Rightarrow \text { Monad }(\text { Free } f) \text { where } \\
& \text { return } a=\text { Return } a \\
& \text { Return } a \ggg f=f a \\
& \text { Free } g \ggg f=\text { Free }(\text { fmap }(\gg f) g)
\end{aligned}
$$

Functions interpreting Free are built using the function fold:

$$
\begin{aligned}
& \text { fold : Functor } f \Rightarrow(a \rightarrow b) \rightarrow(f b \rightarrow b) \rightarrow \text { Free } f a \rightarrow b \\
& \text { fold ret alg }(\text { Return } a)=\text { ret } a \\
& \text { fold ret alg }(\text { Free } f)=\text { alg }(\text { fmap }(\text { fold ret alg }) f)
\end{aligned}
$$

where the first argument ret is applied to pure values and the second argument alg is an algebra that is applied to impure values.

We use the coproduct + of two Functors to express the combination of two effects:

$$
\text { data }(f+g) a=\operatorname{Inl}(f a) \mid \operatorname{Inr}(g a) \text { deriving Functor }
$$

This data type is similar to the Either type, but it works on type constructors (kind $* \rightarrow *$ ) instead of types (kind $*$ ). We use the following two effect signatures to express recursion and non-determinism respectively. Their functor instances are automatically derived by GHC.

$$
\begin{aligned}
& \text { data RecF } i \text { o } a=\operatorname{RecF} i(o \rightarrow a) \text { deriving Functor } \\
& \text { data NDF } a=\operatorname{OrF} \text { a } a \mid \text { FailF } \\
& \text { deriving Functor }
\end{aligned}
$$

For convenience, we label the combined free monad (which is isomorphic to the NDRec type) with a type synonym NDRecF, and define some smart constructors for it:

$$
\begin{aligned}
& \text { type } N D R e c F i o=\text { Free }(N D F+\operatorname{RecF} i o) \\
& \text { orF }:: N D R e c F \text { i o a } \rightarrow \text { NDRecF } i \text { o a } \rightarrow \text { NDRecF } i \text { o a } \\
& \text { orF } l r=\text { Free }(\text { Inl }(\text { OrF l r })) \\
& \text { chooseF }::[a] \rightarrow \text { NDRecF } i \text { o a } \\
& \text { chooseF }=\text { foldr orF failF o map return where } \\
& \quad \text { failF }=\text { Free }(\text { Inl FailF }) \\
& \text { recF }:: i \rightarrow N D R e c F \text { i o o } \\
& \text { recF } i=\text { Free }(\text { Inr }(\text { RecF } i \text { Return }))
\end{aligned}
$$

Effect Handler Implementation for Lattices The typeclass Lattice is defined as follows:

$$
\begin{gathered}
\text { class Lattice } l \text { where } \\
\text { bottom }:: l \\
\text { join } \quad:: l \rightarrow l \rightarrow l
\end{gathered}
$$

This typeclass prioritizes the view of the $\sqcup$-operator as a binary operator because this is the most useful in practice.

Now we replace every FailF in a value of type $N D R e c F$ with bottom and every $\operatorname{OrF}$ with join:

$$
\begin{aligned}
& \text { runND :: (Functor } f \text {, Lattice o) } \\
& \Rightarrow \text { Free }(N D F+f) o \rightarrow \text { Free } f o \\
& \text { runND }=\text { fold return alg where } \\
& \text { alg }(\text { Inl FailF })=\text { return bottom } \\
& \operatorname{alg}(\operatorname{Inl}(\text { OrF l r }))=\text { join }<\$>l<*>r \\
& \operatorname{alg}(\operatorname{Inr} f) \quad=\text { Free } f
\end{aligned}
$$

The effect handler runND effectively eliminates the $N D F$ effect from the syntax tree. Only the recursive effect $\operatorname{RecF} i$ o remains. This effect is then interpreted by runRec:

$$
\begin{aligned}
& \text { runRec }::(\text { Ord } i, \text { Eq o, Lattice o }) \\
& \Rightarrow(i \rightarrow \text { Free }(\text { RecF } i \text { o }) \rightarrow i \rightarrow o \\
& \text { runRec expr } i_{0}=\text { lfp step } s_{0} ! i_{0} \text { where } \\
& \quad=\text { M.singleton } i_{0} \text { bottom } \\
& s_{0} \quad \text { step } m=\text { foldr }(\lambda k \rightarrow \text { go }(\text { expr } k) k) m(\text { M.keys } m) \\
& \text { go expr }=\text { fold onReturn alg expr where } \\
& \text { onReturn } x i=M . \text { insertWith join } i x \\
& \text { alg }(\text { RecF } j k) i m=\text { case } M \text {.lookup } j m \text { of } \\
& \quad \text { Nothing } \rightarrow k \text { bottom } i(M . \text { insert } j \text { bottom } m) \\
& \text { Just } l \rightarrow k l i m
\end{aligned}
$$

Note that runRec does not expect a plain effect, but an effectful function $i \rightarrow$ Free (RecF $i o$ ) $o$, and also returns a function $i \rightarrow$ $o$. Essentially, runRec is a memoizing fixpoint operator applied to the recursive equation defined by expr.

Reconsider the shortest path program from Section 6:

$$
\begin{aligned}
& \text { spF :: Node } \rightarrow \text { Node } \rightarrow \text { NDRecF Node Dist Dist } \\
& \text { spF dst src } \mid \text { src } \equiv \text { dst }=\text { return } 0 \\
& \mid \text { otherwise }=\text { do } n \leftarrow \text { chooseF }(\text { adj src }) \\
& d \leftarrow \text { recF } n \\
& \text { return }(d+1)
\end{aligned}
$$


where the Dist datatype and its instances are defined as:

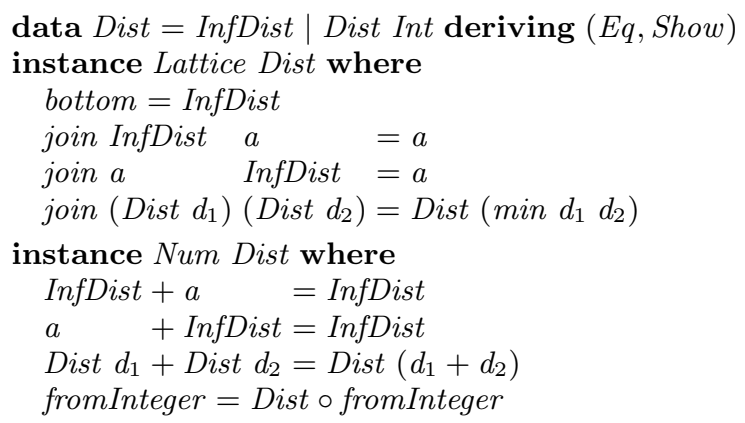

Note that Dist has a Lattice-instance corresponding to the lattice $(\mathbb{N} \cup\{\infty\}, \sqcup)$ from Section 6. Combining runND and runRec gives an effect handler runRec $\circ($ runNDo) implementing the semantic function $\llbracket \cdot \rrbracket_{L}$. For $s p F$ this effect handler computes the expected result:

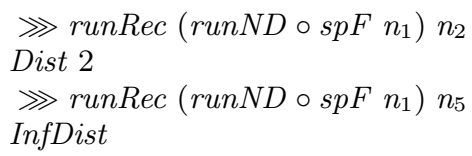

Lifting Continuations The semantic functions $\mathcal{R} \llbracket \cdot \rrbracket$ and $\mathcal{R} \llbracket \cdot \rrbracket_{L}$ only differ in the case for Rec $j k: \mathcal{R} \llbracket \cdot \rrbracket$ computes the union of the $k$ applied to every element of $s(j)$, while $\mathcal{R} \llbracket \cdot \rrbracket_{L}$ directly applies the $k$ to $s(j)$. The similarity between $\mathcal{R} \llbracket \cdot \rrbracket$ and $\mathcal{R} \llbracket \cdot \rrbracket_{\mathcal{P}}(O)$ suggests that we can obtain the behaviour of the former with the latter if we just change the continuation in $\operatorname{Rec} j k$ appropriately. More formally, we want to construct a continuation $k^{\prime}$ such that:

$$
\mathcal{R} \llbracket \operatorname{Rec} j k \rrbracket(s)=\mathcal{R} \llbracket \operatorname{Rec} j k^{\prime} \rrbracket_{\mathcal{P}(O)}(s)
$$

This is achieved by the following code, that lifts a computation in the Free $(N D F+\operatorname{RecF} i o)$-monad to one in the Free $(N D F+$ Rec $i$ (Set o))-monad.

$$
\begin{aligned}
& \text { liftRec }:: \text { Ord o } \\
& \quad \Rightarrow \text { Free }(N D F+\operatorname{RecF} i \text { o }) \text { o } \\
& \rightarrow \text { Free }(\text { NDF }+ \text { RecF } i(\text { Set o }))(\text { Set o }) \\
& \text { liftRec }=\text { fold }(\text { return o singleton }) \text { alg where } \\
& \text { alg }(\text { Inl } f) \quad \text { Free }(\text { Inl } f) \\
& \text { alg }(\text { Inr }(\text { RecF } i k))=\text { Free }\left(\text { Inr }\left(\text { RecF } i k^{\prime}\right)\right) \text { where } \\
& k^{\prime}=\text { fmap unions } \circ \text { traverse } k \circ \text { toList }
\end{aligned}
$$

Since the powerset $\langle\mathcal{P}(O), \cup\rangle$ forms a lattice, we can define a Lattice instance for Set:

$$
\begin{aligned}
& \text { instance } \text { Ord } a \Rightarrow \text { Lattice (Set a) where } \\
& \text { bottom }=\text { empty } \\
& \text { join }=\text { union }
\end{aligned}
$$

Composing liftRec, runND and runRec yields a complete effect handler for Free $(N D F+\operatorname{RecF} i o)$, corresponding to $\llbracket \cdot \rrbracket$ :

$$
\begin{aligned}
& \text { runNDRecF }::(\text { Ord } i, \text { Ord o }) \\
& \Rightarrow(i \rightarrow \text { Free }(N D F+\operatorname{RecF} i o) o) \\
& \rightarrow(i \rightarrow \text { Set o }) \\
& \text { runNDRecF } f=\text { runRec }(\text { runND。liftRec } \circ f)
\end{aligned}
$$

Examples Redefining our running example:

$$
\begin{gathered}
\text { pairF }::() \rightarrow N D R e c F()(\text { Int }, \text { Int })(\text { Int }, \text { Int }) \\
\text { pairF }()=\text { return }(1,2)^{\prime} \text { orF' do }(x, y) \leftarrow \text { recF }() \\
\text { return }(y, x)
\end{gathered}
$$

leads to the following familiar result:
や runRecF pairF ()

fromList $[(1,2),(2,1)]$

To recapitulate, we have shown how to implement the effect handler for $\llbracket \cdot \rrbracket_{L}$ for $N D R e c F$. On top of this we implement the effect handler for $\llbracket \cdot \rrbracket$ for sets, showing that the semantics for lattices generalize the semantics for sets.

\section{MUTUAL RECURSION}

The syntax we have defined so far has a serious limitation: it can only handle a single function at a time. This section lifts this limitation by extending the syntax to support several, potentially mutually recursive functions within the same non-deterministic computation. Moreover, these functions are allowed to possess different argument and return types.

Motivating Example We demonstrate the added power with another grammar analysis. We implement a program that finds the First set of a symbol. A symbol $X$ is in the First set of another symbol $Y$ if any of the strings derived from $Y$ start with $X$. Solving First requires also solving Nullable.

Recall that nullability is characterized by a function from Symbol to Any. First is a also characterized by a function, but one from Symbol to Set Symbol (recall that Set is also a lattice). We encode this fact with a Generalized Algebraic Data Type (GADT) [14]:

$$
\begin{aligned}
& \text { data Analyze o where } \\
& \text { Nullable }:: \text { Symbol } \rightarrow \text { Analyze Any } \\
& \text { First } \quad:: \text { Symbol } \rightarrow \text { Analyze }(\text { Set Symbol })
\end{aligned}
$$

The arguments to the data constructors Nullable and First indicate the argument type (in both cases Symbol). The argument to the type constructor Analyze indicates the return type (Any or Set Symbol).

The syntax data type $N D M$ is a variation of NDRec:

$$
\begin{array}{ll}
\text { data NDM } i & a \text { where } \\
\text { SuccessM } & :: a \rightarrow N D M i a \\
\text { FailM } & :: \text { NDM i a } \\
\text { OrM } & : \text { NDM } i a \rightarrow N D M i a \rightarrow N D M i a \\
\text { RecM } & :: \text { Lattice } o \\
& \Rightarrow i o \rightarrow(o \rightarrow N D M i a) \rightarrow N D M i a
\end{array}
$$

The recursive call constructor RecM expects an existentially qualified parameter $o$ (which must be a lattice). The previous syntax introduces $o$ in the type, and as such fixes $o$ to one particular type for the entire syntax tree. Because of the existential parameter, $N D M$ does allow calls with different return types. Furthermore, the argument is of type $i o$. When $i$ is a GADT, such as Analyze, the type $i o$ will only be inhabited for the desired os (e.g. Any and Set Symbol for Analyze).

We also define four additional smart constructors $\operatorname{rec} M$, choose $M$, choice $M$ and guard $M$. Their meaning is analogous to the smart constructors for NDRec. For brevity, their signature and implementation is omitted here. With these smart constructors we define explicit recursive calls for nullable and first.

$$
\begin{aligned}
& \text { nullable :: Symbol } \rightarrow \text { NDM Analyze Bool } \\
& \text { nullable }=\text { fmap getAny } \circ \text { recM } \circ \text { Nullable } \\
& \text { first }:: \text { Symbol } \rightarrow \text { NDM Analyze Symbol } \\
& \text { first } s=\text { recM (First } s) \ggg \text { choose } M \circ \text { toList }
\end{aligned}
$$

Solving Nullable and First is straightforward:

$$
\text { analyze :: Grammar } \rightarrow \text { Analyze o } \rightarrow \text { NDM Analyze o }
$$$$
\text { analyze } g \text { (Nullable } s \text { ) }
$$ 




In order to determine the First set of a symbol $s$, we need to find the longest nullable prefix of the body for every production with $s$ in the head. Then we find the First sets of every symbol in the prefix and the symbol directly following the prefix.

Implementation From Analyze we derive the actual input and output types that need to be stored in the environment:

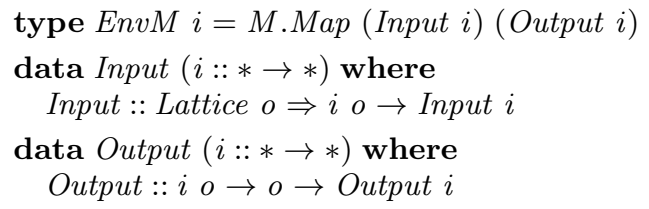

The data types Input and Output wrap a particular instantiation of the Analyze type constructor in an existential quantification. The implementation of the $E q$ and $O r d$ instances is elided for brevity.

We use the typeclass Coerce to type-safely coerce Output Analyze back to Any or Set Symbol, depending on which constructor of Analyze is pattern-matched.

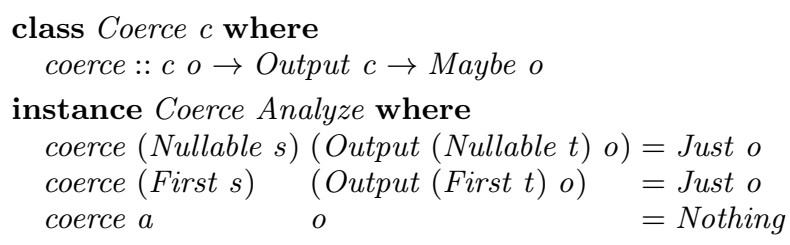

Now we lift join to Outputs:

$$
\begin{gathered}
\text { joinO }::(\text { Coerce } i, \text { Lattice o) } \\
\Rightarrow i \text { Output } i \rightarrow \text { Output } i \rightarrow \text { Output } i \\
\text { joinO } i o_{1} o_{2}= \\
\text { maybe } \left.o_{1} \text { (Output } i\right)\left(\text { join }<\$>\text { coerce } i o_{1}\right. \\
\left.<*>\text { coerce } i o_{2}\right)
\end{gathered}
$$

At last we have all the pieces to define the effect handler. The function $g o M$ evaluates an NDM $i$ o, given an environment EnvM $i$ and runNDM computes the least fixed point of $g o M$ :

$$
\begin{aligned}
& \text { goM }::(\text { Coerce } i, \text { Lattice o, Ord }(\text { Input } i)) \\
& \quad \Rightarrow i \text { o NDM } i \text { o } \rightarrow \text { EnvM } i \rightarrow \text { EnvM } i \\
& \text { goM } i(\text { SuccessM } x) m= \\
& M \text {.insertWith }(\text { joinO } i) \text { (Input } i) \text { (Output } i x) m \\
& \text { goM } i \text { FailM } \quad m=m \\
& \text { goM } i(\text { OrM } l r) \quad m=\text { goM } i r(\text { goM } i l m) \\
& \text { goM } i(\text { RecM } j k) \quad m= \\
& \text { case M.lookup }(\text { Input } j) m \gg \text { coerce } j \text { of }
\end{aligned}
$$

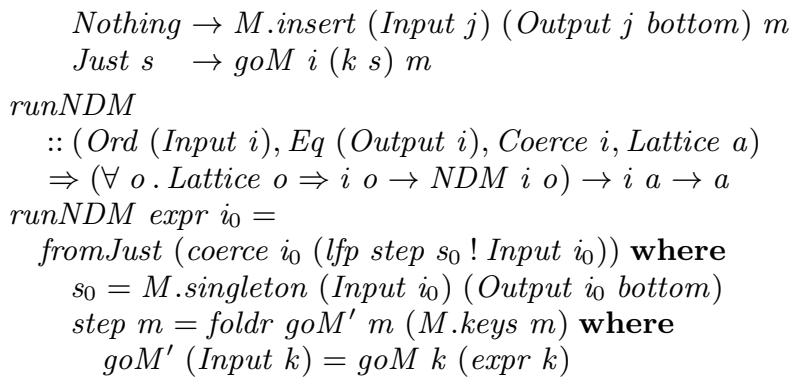

This implementation is not very different from runNDRec (see Section 4), except that some wrapping and unwrapping of Input and Output is required. Note that dependency tracking is orthogonal to the problem of mutual recursion, i.e. dependency tracking is easily added.

Evaluation Using runNDM we now solve First, which requires solving Nullable, in the same computation.

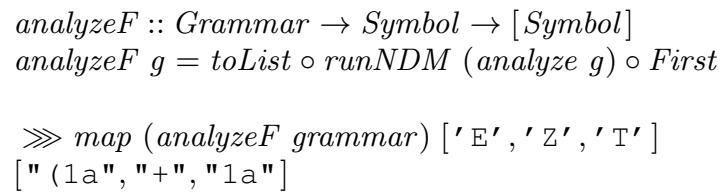

The computed First sets of E,Z and $\mathrm{T}$ are the results one would expect from the definition: an expression may start with a parenthesis, a literal or an identifier, and similarly for sub-expressions and terms. We now extend First to strings of symbols:

$$
\begin{aligned}
& \text { first } S: \text { Grammar } \rightarrow[\text { Symbol }] \rightarrow \text { Set Symbol } \\
& \text { firstS } \mathrm{g} s= \\
& \text { let nulls }=\text { takeWhile analyzeN } s \\
& \text { notNulls }=\text { drop While analyzeN } s \\
& \text { prefix }=\text { nulls }+ \text { take } 1 \text { notNulls } \\
& \text { analyzeN }=\text { getAny } \circ \text { runNDM }(\text { analyze g) } \circ \text { Nullable } \\
& \text { in unions } \$ \text { map (runNDM (analyze g) } \circ \text { First) prefix }
\end{aligned}
$$

So called left-recursive conflicts occur when the First sets of bodies of two rules with the same head have a non-empty intersection. The following function has Conflict uses firstS of rule bodies to detect conflicts:

$$
\begin{aligned}
& \text { hasConflict : Grammar } \rightarrow \text { Bool } \\
& \text { hasConflict } g= \\
& \text { let } \text { ruleHead }(h \longmapsto-)=h \\
& \quad \text { ruleBody }(-\longmapsto b)=b \\
& \quad \text { ruleConflict }[r]=\text { False } \\
& \quad \text { ruleConflict rs }=\neg(\text { null }(\text { foldr } 1 \text { intersection firsts })) \\
& \quad \text { where firsts }=\text { map }(\text { firstS } g \circ \text { ruleBody }) \text { rs } \\
& \text { in or } \$ \text { map ruleConflict } \$ \text { groupBy }((\equiv) \text { 'on' ruleHead }) g
\end{aligned}
$$

Our example grammar is conflict free:

$$
\text { ゆ has Conflict grammar }
$$

False

However, in the following grammar the rules for ' $E$ ' do conflict:

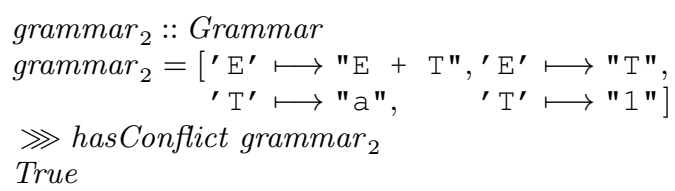

Note that the implementation of firstS and has Conflict is not very efficient: in between calls to runNDM the results are simply thrown away. We see two ways to resolve this: either have 


\begin{tabular}{lrrr}
\hline & Size & Naive & $\begin{array}{l}\text { Dependency } \\
\text { Tracking }\end{array}$ \\
\hline Fibonacci & 800 & 455.7 & 13.77 \\
& 805 & 462.8 & 13.85 \\
& 810 & 469.3 & 13.97 \\
\hline Knapsack & 10 & 155.9 & 8.185 \\
& 15 & 226.6 & 12.32 \\
& 20 & 309.0 & 16.04 \\
\hline NQueens & 7 & 4.864 & 1.259 \\
& 9 & 152.9 & 26.79 \\
& 10 & 914.8 & 132.0 \\
\hline SCC & 30 & 57.31 & 8.843 \\
& 33 & 104.9 & 10.67 \\
\hline Shortest Path & 8000 & 97.97 & 12.67 \\
& 8500 & 1366 & 676.6 \\
& 9000 & 1451 & 718.7 \\
\hline
\end{tabular}

Table 2: The benchmark results in milliseconds.

runNDM return the environment EnvM to directly perform lookups, or embed firstS and hasConflict in $N D M$ as well. Such an embedding requires extending Analyze with new constructors for FirstS and HasConflict. This is left as an exercise to the reader. In a more generic setting, one could imagine using a "data types à la carte"-approach to solve this extension more satisfactorily.

\section{BENCHMARKS}

To demonstrate the performance impact of dependency tracking, we evaluate several benchmarks with and without dependencytracking effect handlers and compare their results.

We consider five problems: computing (large) fibonacci numbers, (a variation of) the knapsack problem, the nqueens problem, computing strongly connected components in a directed graph and finding the shortest path in a directed graph.

The benchmarks were build using the Criterion-benchmark harness and compiled using GHC 7.10.2 (with optimization setting -02) on a 64-bit Linux machine with an Intel Core i7-2600 @ 3.6 Ghz and 16 GB of RAM. All code and results are available in the the Bitbucket repository. ${ }^{10}$

Table 2 shows the ordinary least squares regression of all samples of the execution time. The $R^{2}$ goodness of fit was above 0.99 in all cases. All values are in milliseconds.

For Fibonacci the first column is the index of the fibonacci number that is computed. For Knapsack it specifies the number of items to choose from (the capacity of the knapsack is fixed to 200). For NQueens, it indicates the number of queens that need to be placed on the board. For SCC it is the number of nodes in the graph (the number of edges is fixed to half the number of possible edges). Finally, for Shortest Path it is the number of edges in the graph.

The results show that effect handlers with dependency tracking massively outperform the naive effect handlers.

\section{RELATED WORK}

There are various related works.

Least fixed point computations In their seminal work [17] Scott and Strachey have introduced the idea of denotational mathematical semantics. In particular, they already define the semantics of programming language syntax based on least fixed points.

\footnotetext{
${ }^{10} \mathrm{https} / / /$ bitbucket.org/AlexanderV/thesis, in the IFL2015/benchmarks directory.
}

Jeannin et al. [8] give a category theoretic treatment of "Nonwell-Founded computations". They give many examples, several of which can be implemented in our non-deterministic framework. Others are beyond our iterative least fixed point solver, and require more sophisticated techniques, for instance, Gaussian elimination.

The use of least fixed points for solving grammar analysis problems has been extensively treated by Jeuring and Swierstra [9].

It is a well-known result that dynamic programming can be viewed as memoization of recursive equations. The related work section of Swadi et al. gives an overview [19].

Effect Handlers Algebraic effects as a way to write effectful programs are the subject of much contemporary research $[27,10]$.

New languages have been created specifically for studying algebraic effect handlers: Eff [1] extends ML with syntax constructs for effect handlers and Frank [12] which has a Haskell-like syntax and tracks effect in its type system.

Non-determinism The classical introduction of non-determinism in functional programming based on the list monad is due to Wadler [24]. Hinze [7] derives a backtracking monad transformer from a declarative specification. This transformer adds backtracking (non-determinism and failure) to any monad.

Kiselyov et al. [11] improve on Hinze's work by implementing backtracking monad transformers which support fair non-deterministic choice. They present three different implementations: the classical approach based on Streams (i.e. lazy lists), one based on continuation passing style and another one based on control operators for delimited continuations.

Tabling in Prolog Since Prolog has native non-determinism, it too suffers from the issues we discussed in the introduction: nondeterminism and recursion can interact to cause non-termination even when a finite solution does exist. The semantics of definite logic programs is given by the least fixed point of the immediate consequence operator $T_{P}[23]$ that strongly resembles the semantics given by $\llbracket \cdot \rrbracket$. Tabling is a technique that produces a different semantics in the same way we produce different semantics for non-determinism. Several Prolog implementations support tabling, such as XSB-Prolog [22, 26], B-Prolog [28], YapProlog [15], ALS-Prolog [5] and Mercury [18]. XSB-Prolog in particular also implements lattice-based answer subsumption [21] which strongly resembles and has partially inspired the techniques of Section 6. Other systems provide similar functionality through mode-directed tabling [16, 29,6].

Explicit Recursion We are not the first to tame unbridled recursion by representing recursive calls explicitly. Devriese and Piessens $[3,4]$ overcome the limitations of so-called " $\omega$-regular" grammars by representing recursive occurrences of non-terminals in production bodies explicitly.

McBride [13], working in the dependently typed total language Agda, ${ }^{11}$ gives a free monadic model for recursive calls. Several possible semantics are discussed, but none based on fixed points.

Oliveira and Cook [2] extend traditional algebraic datatypes to with explicit definition and manipulation of cycles, offering a practical and convenient way of programming graphs in Haskell. Their approach to explicating recursion differs from ours. Where we use the smart constructor rec to indicate a recursive call, they instead use open recursion. While this is a good fit for the explicit graph data structure they work with, for our purposes (non-deterministic computations) the former style is more convenient.

\section{CONCLUSIONS}

We have described mutually recursive non-deterministic compu-

\footnotetext{
${ }^{11}$ http://wiki.portal.chalmers.se/agda/pmwiki.php
} 
tations in a free monadic fashion, and have given these descriptions a concise denotational semantics in terms of sets, and more generally in terms of lattices. We have shown how to efficiently implement these semantics in Haskell using Effect Handlers.

\section{ACKNOWLEDGEMENTS}

We thank Maciej Piròg for pointing out related work, and Georgios Karachalias, Steven Keuchel, Amr Saleh and Paolo Torrini, Maarten Van den Heuvel and the IFL 2015 participants for their helpful feedback. We are grateful to the anonymous reviewers of the IFL 2015 PC commitee for their useful comments. This research was partially supported by the Flemish Fund for Scientific Research (FWO). This work was partially supported by project GRACeFUL, which has received funding from the European Union's Horizon 2020 research and innovation programme under grant agreement No 640954.

\section{REFERENCES}

[1] A. Bauer and M. Pretnar. Programming with algebraic effects and handlers. Journal of Logical and Algebraic Methods in Programming, 84(1):108 - 123, 2015. Special Issue: The 23rd Nordic Workshop on Programming Theory (NWPT 2011) Special Issue: Domains X, International workshop on Domain Theory and applications, Swansea, 5-7 September, 2011.

[2] B. C. d. S. Oliveira and W. R. Cook. Functional programming with structured graphs. In ICFP, pages 77-88. ACM, 2012.

[3] D. Devriese and F. Piessens. Explicitly recursive grammar combinators. In Practical Aspects of Declarative Languages, PADL, volume 11, pages 84-98. Springer, 2011.

[4] D. Devriese and F. Piessens. Finally tagless observable recursion for an abstract grammar model. Journal of Functional Programming, 22:757-796, 2012.

[5] H.-F. Guo and G. Gupta. A simple scheme for implementing tabled logic programming systems based on dynamic reordering of alternatives. In Proceedings of the 17th International Conference on Logic Programming, pages 181-196. Springer-Verlag, 2001.

[6] H.-F. Guo and G. Gupta. Simplifying dynamic programming via mode-directed tabling. SoftwarePractice \& Experience, 38(1):75-94, 2008.

[7] R. Hinze. Deriving backtracking monad transformers. In In The International Conference on Functional Programming (ICFP), 2000.

[8] J.-B. Jeannin, D. Kozen, and A. Silva. Language constructs for non-well-founded computation. In Programming Languages and Systems, volume 7792 of Lecture Notes in Computer Science, pages 61-80. Springer Berlin Heidelberg, 2013.

[9] J. Jeuring and D. Swierstra. Constructing functional programs for grammar analysis problems. In Proceedings of the seventh international conference on Functional programming languages and computer architecture, pages 259-269. ACM, 1995.

[10] O. Kiselyov, A. Sabry, and C. Swords. Extensible Effects: An alternative to monad transformers. In Proceedings of the 2013 ACM SIGPLAN Symposium on Haskell, Haskell '13, pages 59-70, New York, NY, USA, 2013. ACM.

[11] O. Kiselyov, C.-c. Shan, D. P. Friedman, and A. Sabry. Backtracking, interleaving, and terminating monad transformers:(functional pearl). In ACM SIGPLAN Notices, volume 40, pages 192-203. ACM, 2005.

[12] C. McBride. The Frank Manual, 2012.

[13] C. McBride. Turing-completeness totally free. In Mathematics of Program Construction, volume 9129 of Lecture Notes in Computer Science, pages 257-275. Springer International Publishing, 2015.

[14] S. Peyton Jones, D. Vytiniotis, S. Weirich, and G. Washburn. Simple unification based type inference for GADTs. In In The International Conference on Functional Programming (ICFP), 2006.

[15] R. Rocha, F. Silva, and V. Santos Costa. YapTab: A tabling engine designed to support parallelism. In Conference on Tabulation in Parsing and Deduction, pages 77-87, 2000.

[16] J. Santos and R. Rocha. Efficient support for mode-directed tabling in the YapTab Tabling System. CICLOPS 2012, page 41, 2012.

[17] D. Scott and C. Strachey. Toward a mathematical semantics for computer languages, volume 1. 1971.

[18] Z. Somogyi and K. Sagonas. Tabling in Mercury: design and implementation. In Proceedings of the 8th international conference on Practical Aspects of Declarative Languages, pages 150-167. Springer-Verlag, 2006.

[19] K. Swadi, W. Taha, O. Kiselyov, and E. Pasalic. A monadic approach for avoiding code duplication when staging memoized functions. In Proceedings of the 2006 ACM SIGPLAN Symposium on Partial Evaluation and Semantics-based Program Manipulation, PEPM '06, pages 160-169, New York, NY, USA, 2006. ACM.

[20] W. Swierstra. Data types à la carte. Journal of functional programming, 18(04):423-436, 2008.

[21] T. Swift and D. S. Warren. Tabling with answer subsumption: Implementation, applications and performance. In Logics in Artificial Intelligence, pages 300-312. Springer, 2010.

[22] T. Swift and D. S. Warren. XSB: Extending Prolog with tabled logic programming. Theory and Practice of Logic Programming, 12(1-2):157-187, 2012.

[23] M. H. Van Emden and R. A. Kowalski. The Semantics of Predicate Logic As a Programming Language. J. ACM, 23(4):733-742, Oct. 1976.

[24] P. Wadler. How to replace failure by a list of successes a method for exception handling, backtracking, and pattern matching in lazy functional languages. In Functional Programming Languages and Computer Architecture, pages 113-128. Springer, 1985.

[25] P. Wadler. Monads for functional programming. In Advanced Functional Programming, pages 24-52. Springer, 1995.

[26] D. S. Warren. Programming in Tabled Prolog, volume 1. http://www3.cs.stonybrook.edu/ warren/xsbbook/, 1999.

[27] N. Wu and T. Schrijvers. Fusion for free - efficient algebraic effect handlers. In Mathematics of Program Construction 12th International Conference, MPC 2015, Königswinter, Germany, June 29 - July 1, 2015. Proceedings, pages 302-322, 2015.

[28] N.-F. Zhou. The language features and architecture of B-Prolog. Theory and Practice of Logic Programming, 12(1-2):189-218, 2012.

[29] N.-f. Zhou, Y. Kameya, and T. Sato. Mode-directed tabling for dynamic programming, machine learning, and constraint solving. In Proceedings of the International Conference on Tools with Artificial Intelligence (ICTAI), 2010. 